\title{
Thermally Induced Gelation of Mixed Phosphatidylcholine Aqueous Solution Containing Wormlike Micelle Structure
}

\author{
Natdanai FAFAUNGWITHAYAKUL ${ }^{1}$, Utai KLINKESORN ${ }^{2}$, Tom BRENNER ${ }^{1}$, \\ Nutsuda VICHAKACHARU ${ }^{2}$, Shingo MATSUKAWA ${ }^{1, \dagger}$ \\ ${ }^{1}$ Graduate School of Science and Technology, Tokyo University of Marine Science and Technology, \\ 4-5-7 Konan, Minato-ku, Tokyo 108-8477, JAPAN \\ ${ }^{2}$ Deparment of Food Science and technology, Faculty of Agro-Industry, Kasetsart University, \\ 50 Ngam Wong Wan Road, Ladyaow, Chatuchak, Bangkok 10900, THAILAND
}

\begin{abstract}
We report for the first time on wormlike micelle formation in aqueous mixtures of lysophosphatidylcholine (LPC) and phosphatidylcholine (PC). The viscosity of mixtures of these two phospholipids at total concentrations $\geq 56 \mathrm{mM}$ showed a marked maximum around LPC molar fraction $0.5-0.7$, which was ascribed to wormlike micelle formation. The diffusion coefficient of the lecithin, as measured using pulsed-field-gradient stimulated spin echo NMR, suggests that LPC and PC form spherical micelles and vesicle structures, respectively. In mixtures of LPC and PC, individual spherical micelles of LPC and a vesicle structure of PC were found at low temperature. Wormlike micelle formation was manifested in a steep decrease of the diffusion coefficient at temperatures above $55^{\circ} \mathrm{C}$. The results indicate that a minimum concentration of both LPC and PC, as well as a molar ratio of LPC to PC close to $0.5-0.6$, are prerequisite for thermally induced formation of lecithin wormlike micelles in aqueous solutions. Following formation on heating, the wormlike micelles remained stable on cooling, and no population of smaller spherical micelles could be detected.
\end{abstract}

Keywords: lecithin, wormlike micelles, phospholipids, gradient NMR, diffusion coefficient

\section{Introduction}

An increasing amount of research effort is being directed towards wormlike micelles. Wormlike micelles are one structure formed by surfactant self-assembly. This structure is characterized by long flexible chains. The dynamics of wormlike micelle suspensions can be described using a single (reptational) relaxation process [1]. Consequently, the rheology of these suspensions can be described using a simple Maxwell fluid model [1-2]. Characteristic relaxation times and zero shear-rate viscosities reported for dilute or semi-dilute $(<10 \% \mathrm{w} / \mathrm{w})$ wormlike micelle aqueous suspensions are in the range 0.1-100 s and $0.1-10^{3}$ Pa.s, respectively $[1,3,4]$.

Wormlike micelles can be formed in either non-polar organic solvents, where reverse wormlike micelles are observed, or in water, where they can also be called normal wormlike micelles. Wormlike micelles can be formed using different routes, such as application of ultraviolet/ visible light [3] and changes in temperature [5] or $\mathrm{pH}$

(Received 13 Jun. 2014: accepted 10 Aug. 2014)

† Fax: 03-5463-0581, E-mail: matsukaw@kaiyodai.ac.jp
[6]. Wormlike micelles have been considered for applications in different fields, including drug delivery systems $[7,8]$, personal care products [9] and biosensors [10, 11]. In water, most studies have concentrated on ionic surfactants such as cetyltrimethylammonium bromide (CTAB) and cetyltrimethylammonium 3-hydroxynaphthalene 2-carboxylate (CTAHNC), which form wormlike micelles on addition of salt $[5,6,12,13]$. In non-polar solvents, on the other hand, formation of reverse lecithin wormlike micelles has been observed on addition of water $[3,4,14,15]$.

Lecithin is the common name applied either to a mixture of phospholipids found in egg yolk and soybean in high concentrations, or strictly to the main member of this mixture, phosphatidylcholine. Phosphatidylcholine, hereafter PC, is a glycerol esterified with two moles of fatty acid, while the third glycerol oxygen is phosphorylated with a choline residue. The length and doublebond content of the fatty acids vary depending on the source. It is one of the major components of the cell membrane. Bilayer structure has therefore been studied as a model of cell membranes. Lysophosphatidylcholine, hereafter LPC, obtained through hydrolysis of phosphati- 
dylcholine by enzyme phospholipase $\mathrm{A}_{2}$, has one mole of fatty acid per mole of phospholipid in position $s n^{-1}\left[16^{-}\right.$ 19]. The use of lecithin as a stabilizer of $\mathrm{w} / \mathrm{o}$ and $\mathrm{o} / \mathrm{w}$ emulsions and its possible applications in the food, pharmaceutical and medical care industries have been reviewed recently [20].

Surfactant self-assemblies are usually related to the critical packing parameter $(C P P): C P P=v / a_{0} l$, where, where $v$ is the volume of the hydrophobic chain, $l$ is the effective length of the hydrophobic chain and $a_{0}$ is the effective surface area of the head-group [21]. The $C P P$ of LPC is about 0.34 , and LPC tends to form spherical micelles [22]. As noted above, PC has two alkyl chains and therefore a higher $C P P$ (around 0.74). As a result, it tends to form planar or vesicle structures [22].

Our hypothesis was that mixtures of PC and LPC could display a different self-assembly than the individual phospholipids. Therefore, we focused on the phase behavior of LPC/PC mixtures in aqueous solution. The effect on the bulk properties was studied with fallingball measurements of the viscosity. The self-assembly behavior was studied using pulsed field gradient nuclear magnetic resonance, hereafter PFG-NMR, which is used to measure the self-diffusion coefficient of molecules and macromolecular aggregates. Application of PFGNMR to surfactant solutions [23, 24] and other multicomponent systems [25] yields weight-averaged information on the distribution of diffusion coefficients. Dynamic light scattering (DLS), on the other hand, yields $\mathrm{Z}^{-}$-averaged distributions of diffusion coefficients. PFG-NMR NMR can therefore be more suitable than DLS in characterization of multi-component samples, especially when the main interest is in the behavior of the smaller macromolecules [24]. In this study, we elucidated the effect of temperature and content of PC and LPC on the formation of wormlike micelles. A better understanding of the factors determining formation of lecithin wormlike micelles could contribute to the design of suitable self-assembly structures for applications in food, drug delivery or bio- mimetic materials. Mixtures of uncharged phospholipids could be an especially suitable choice in some emulsified formulations, as they have been shown to confer higher stability against droplet flocculation compared with charged phospholipids in the presence of small amounts of $\mathrm{NaCl}$ or $\mathrm{CaCl}_{2}$ [26-28].

\section{Materials and Methods}

\subsection{Materials}

Egg yolk PC and LPC (Fig. 1) were kindly provided by Kewpie Company (Tokyo, Japan). According to information from the manufacturer, majority of alkyl chains were C-18 (57.5\%) and C-16 (33.2\%). Deionized water was obtained by a Water Purifier (Autostill WA500, Yamato Scientific Co., LTD., Tokyo, Japan). $\mathrm{D}_{2} \mathrm{O}$ (deuterium isotopic content: 99.0\%) was purchased from Kanto Chemical Co., Inc. (Tokyo, Japan).

PC and LPC were dispersed in deionized water with a sonicator (Qsonica Q125, Connecticut, United States) for $5 \mathrm{~min}$. An ice bath was used to prevent overheating of the sample. The phospholipid dispersions were mixed to obtain mixtures of $\mathrm{C}_{\mathrm{T}}$ (total lecithin concentration; $\mathrm{C}_{\mathrm{T}}=$ $\mathrm{C}_{\mathrm{LPC}}+\mathrm{C}_{\mathrm{PC}}=$ molar concentration of $\mathrm{LPC}+$ molar concentration of $\mathrm{PC}$ ) in the range $8-72 \mathrm{mM}$, and sonicated for 1 min. Sonication was performed at $20 \mathrm{kHz}$, amplitude $60 \%$, and a time interval of $5 \mathrm{~s}$. Solutions used in NMR measurements were prepared with $\mathrm{D}_{2} \mathrm{O}$ instead of deionized water.

\subsection{Methods}

\subsubsection{Light microscopy}

The structure of individual LPC and PC dispersions was observed at room temperature using a light microscope (Olympus model BX 53; Olympus, US).

\subsubsection{Viscosity measurements}

The viscosity of lecithin mixtures was measured with the falling ball method [29]. A coated steel ball was placed in a $20 \mathrm{~cm}$ glass tube filled with sample. Sample
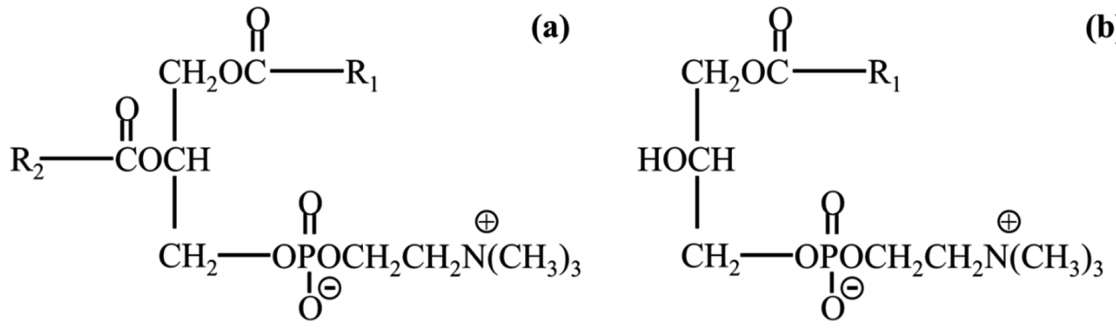

(b)

Fig. 1 Chemical structure of phosphatidylcholine (PC; a) and lysophosphatidylcholine (LPC; b); $\mathrm{R}_{1}, \mathrm{R}_{2}=$ alkyl chains. 
tubes were placed in a temperature-controlled water bath. The time $(t)$ required for the coated steel ball to fall through the glass tube was measured, and the viscosity was calculated using deionized water as a reference:

$$
\eta=\eta_{0} \frac{t}{t_{0}}
$$

where $\eta$ is the viscosity, $\eta_{0}$ is the viscosity of $\mathrm{H}_{2} \mathrm{O}$ and $t_{0}$ is the falling time in $\mathrm{H}_{2} \mathrm{O}$.

\subsubsection{Diffusion measurements}

Diffusion coefficients were measured using the pulsed field gradient stimulated spin-echo (PFG-STE) pulse sequence on a Bruker Avance II 400WB spectrometer (Bruker Corp., US) equipped with a gradient probe [30, 31]. In the PFG-STE pulse sequence, the intervals between the first and second $\left(\tau_{1}\right)$ and the second and third $\left(\tau_{2}\right)$ r.f. pulses were $1 \mathrm{~ms}$ and $10 \mathrm{~ms}$, respectively. The gradient pulse duration $\delta$ and the interval time of the field-gradient-pules $\Delta$ were $1 \mathrm{~ms}$ and $10 \mathrm{~ms}$, respectively. The gradient field strength $g$ was varied in 8 steps in the range $100-1200$ or $100-1500 \mathrm{G} / \mathrm{cm}$. The diffusion coefficient was determined using the following relation between the echo signal intensity and field-gradient parameters:

$$
I(\mathrm{~g})=I(0) \exp \left[-\gamma^{2} \delta^{2} g^{2}<z^{2}>/ 2\right]
$$

where $I(\mathrm{~g})$ and $I(0)$ are echo signal intensities at $t=$ $2 \tau_{2}+\tau_{1}$ with and without the field gradient pulse, respectively. $\gamma$ is the gyromagnetic ratio of ${ }^{1} \mathrm{H} .\left\langle z^{2}\right\rangle$ is the mean square displacement in the $z$ direction (the direction of $P F G$ ) and is equal to $2 D T_{d}$ for normal diffusion, where $D$ is the diffusion coefficient and the diffusion time $T_{\mathrm{d}}$ for a square-shaped gradient in the PFG-STE pulse is $\Delta-\delta / 3$. Therefore, eq. (2) is rewritten as:

$$
I(\mathrm{~g})=I(0) \exp \left[-\gamma^{2} \delta^{2} g^{2}(\Delta-\delta / 3) D\right]
$$

The temperature was controlled using a Bruker BVT3200 temperature unit and varied from $25^{-}-75^{\circ} \mathrm{C}$. The temperature in the sample tube was measured with an optical fiber thermometer (Takaoka Electric Mfg. Co., Tokyo, Japan).

\section{Results and Discussion}

\subsection{Phase diagram}

The viscosity of lecithin mixtures was used to construct phase diagrams at different temperatures. The concentration of LPC and PC was varied in the range 0-48 mM. At $25^{\circ} \mathrm{C}$, mixtures of LPC and PC showed low viscosity and turbidity at all tested concentrations. LPC solutions were transparent, and their viscosity was below $2 \mathrm{mPa} \cdot \mathrm{s}$. Conversely, PC solutions were turbid, but their viscosity was also below $2 \mathrm{mPa} \cdot \mathrm{s}$. We expected the turbidity of PC solutions to reflect the presence of large self-assembled structures, either vesicle or planar micelles. In Fig. 2, we present the results of light microscopy. As seen from Fig. 2a, no features could be identified for the individual LPC dispersion. As seen from Fig. $2 \mathrm{~b}$, however, large roughly micelles with a diameter of about $2 \mu \mathrm{m}$ were observed in the individual PC dispersion.

Our preliminary tests revealed a marked increase in the apparent viscosity and reduced turbidity of many solutions on heating above $\approx 50^{\circ} \mathrm{C}$. For this reason, more detailed studied were carried out at $55^{\circ} \mathrm{C}$ and $75^{\circ} \mathrm{C}$. The phase diagrams of the lecithin mixtures at $55^{\circ} \mathrm{C}$ and $75^{\circ} \mathrm{C}$ are shown in Fig. 3. An arbitrary threshold of $50 \mathrm{mPa} \cdot \mathrm{s}$ was chosen to represent (apparent) gelation. This apparent gelation is closely related to formation of very large

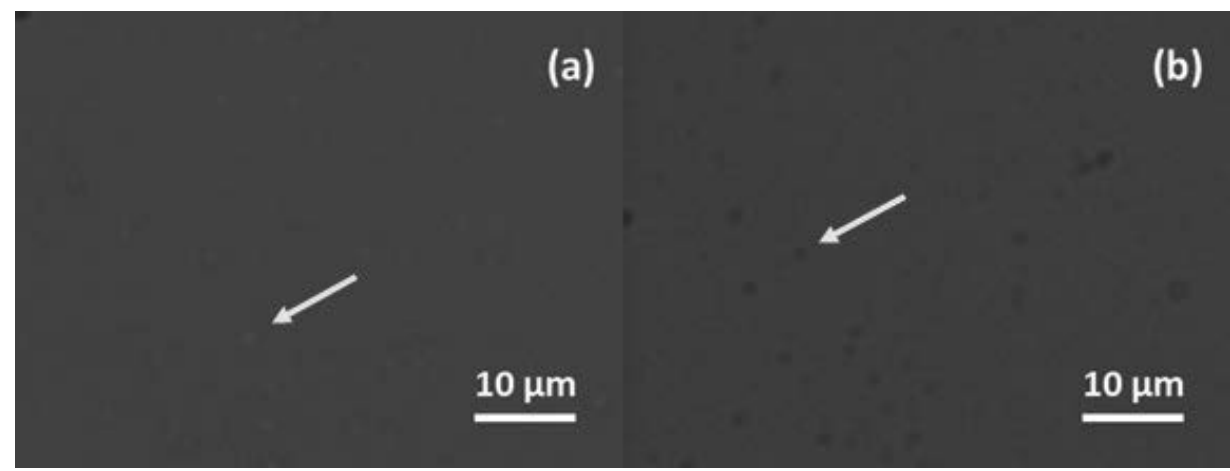

Fig. 2 Micrograph of LPC (a) and PC (b) at room temperature. Bright circles, indicated with an arrow in $2 \mathrm{a}$, are artifacts from entrapped air bubbles. Dark circles, indicated with an arrow in $2 \mathrm{~b}$, are large surfactant assemblies. 


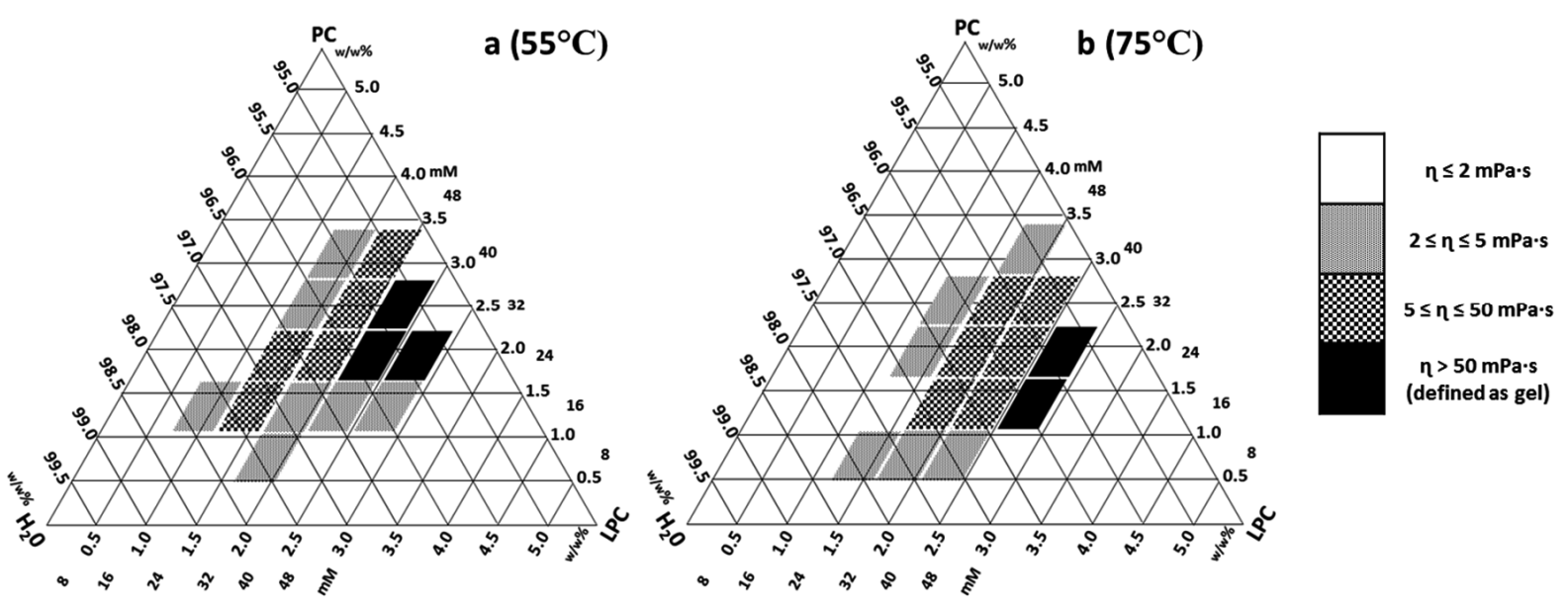

Fig. 3 Partial phase diagram of lecithin mixtures, defined according to their viscosity, at $55^{\circ} \mathrm{C}$ (a) and $75^{\circ} \mathrm{C}$ (b).

mixed micelles, as will be shown later in section 3.2. At $55^{\circ} \mathrm{C}$, the viscosity of individual solutions of LPC and PC remained low for all concentrations tested (Fig. 3a). Mixtures of the two phospholipids, however, showed a markedly increased viscosity, especially mixtures where the concentration of both LPC and PC was above $24 \mathrm{mM}$. In addition, the turbidity of mixtures decreased markedly around $55^{\circ} \mathrm{C}$.

Figure 4 shows the viscosity of PC/LPC mixtures at $55^{\circ} \mathrm{C}$ as a function of the LPC molar fraction, $f_{\mathrm{LPC}}\left(f_{\mathrm{LPC}}=\right.$ $\mathrm{C}_{\mathrm{LPC}} / \mathrm{C}_{\mathrm{T}}$ ). For $f_{\mathrm{LPC}} \approx 0.5-0.7$, mixtures with $\mathrm{C}_{\mathrm{T}} \leq 48 \mathrm{mM}$ showed a maximum, and for $\mathrm{C}_{\mathrm{T}} \geq 56 \mathrm{mM}$ we observed gelation for the same values of $f_{\text {LPC }}$. These results suggest formation of wormlike micellar structures. Davies $e t$ al. [5], who studied mixtures of CTAB and 5-methyl salicylic acid, formation of wormlike micelles leads to a decrease in turbidity and an increase of the viscosity.
This is the first time, to our knowledge, that wormlike micelle formation is reported in aqueous solutions of lecithin without addition of salts. Formation of wormlike micelles has been reported by Arleth et al. [32] for a mixture of PC with the bile salt sodium glycochenodeoxycholate. They noted formation of wormlike micelles only at low concentrations of the bile salt close to its critical micellization concentration, noting that this trend is opposite to the usual trend in micellar systems, where the extent of aggregation is enhanced by increasing the surfactant concentration. Our findings are different, as a minimum content of both LPC and PC is required for wormlike micelle formation. Shumilina et al. [15] reported formation of reverse $\mathrm{PC}$ wormlike micelles in $n$-decane mixtures on addition of a small amount of water. The reverse wormlike micelles disintegrated on addition of LPC. Our findings are, as expected, opposite

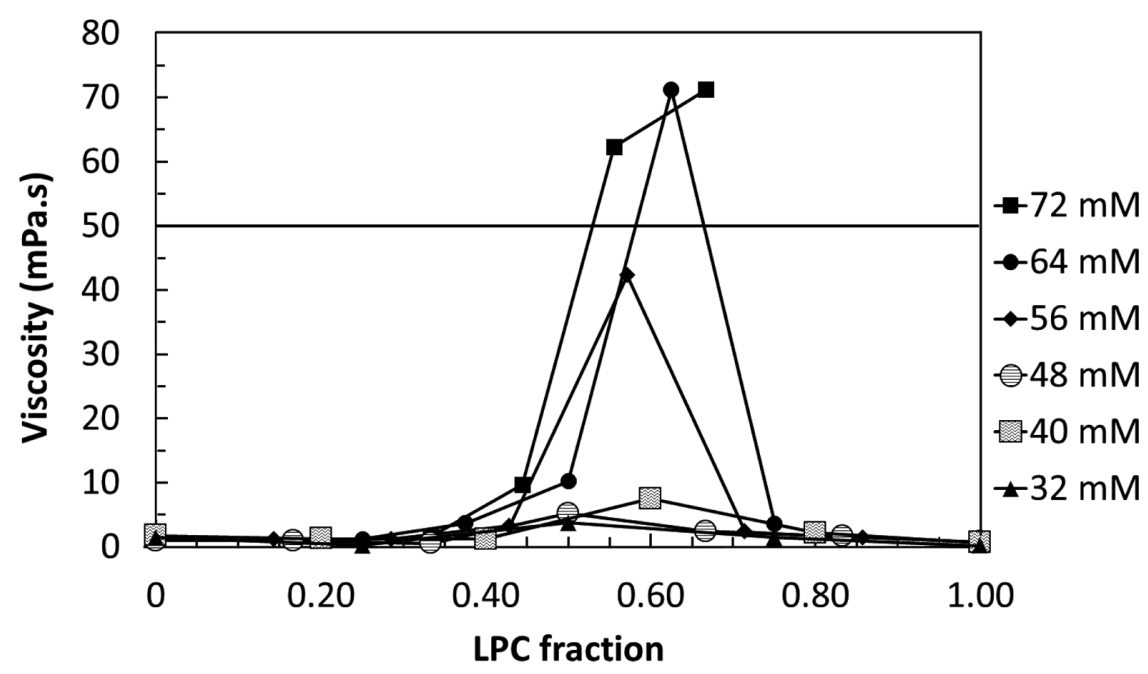

Fig. 4 Viscosity of lecithin mixtures as a function of $\mathrm{f}_{\mathrm{LPC}}$ at $55^{\circ} \mathrm{C}$. The horizontal line indicates apparent gelation $(50 \mathrm{mPa} \cdot \mathrm{s})$. The total lecithin content $\mathrm{C}_{\mathrm{T}}$ is indicated in the figure. 
to theirs. We find that the LPC is essential for formation of the normal lecithin wormlike micelles in aqueous solutions.

The viscosity of lecithin mixtures is plotted as a function of temperature in Fig. 5. For mixtures $\mathrm{C}_{\mathrm{T}}=48 \mathrm{mM}$ (Fig. 5a-c), all samples had viscosities below $10 \mathrm{mPa} \cdot \mathrm{s}$ throughout the heating process. At these concentrations, we propose that the mixtures form relatively short wormlike micelles. As explained by Cates [1], the equilibrium length of the wormlike micelle is expected to increase with increasing surfactant concentration. On subsequent cooling, however, the viscosity of mixtures with $f_{\mathrm{LPC}}$ above 0.5 increased on below about $50^{\circ} \mathrm{C}$. This increase probably reflected an increase in the equilibrium length of the wormlike micelle, as explained by Cates [1], due to the effect of temperature on scission reactions of the chains. Tung et al. [4] studied reverse wormlike micelles formed by lecithin in n-decane/water system and normal wormlike micelles formed by cetylpyridinium bromide and sodium salicylate in water, and studied the effect of temperature only following extensive heating, i.e., after formation of the wormlike micelles was complete. They reported in both cases a marked increase in zero-shear rate viscosity with decreasing temperature, and ascribed it to the increase in equilibrium length of the wormlike micelles with decreasing temperature. For mixtures with $\mathrm{C}_{\mathrm{T}}=64 \mathrm{mM}$ (Fig. 5d-f), the viscosity for $f_{\mathrm{LPC}}=0.5$ increased slightly and for $f_{\mathrm{LPC}}=0.63$ the viscosity increased steeply on heating above $50^{\circ} \mathrm{C}$, suggesting the formation of short and long wormlike micelles, respec-
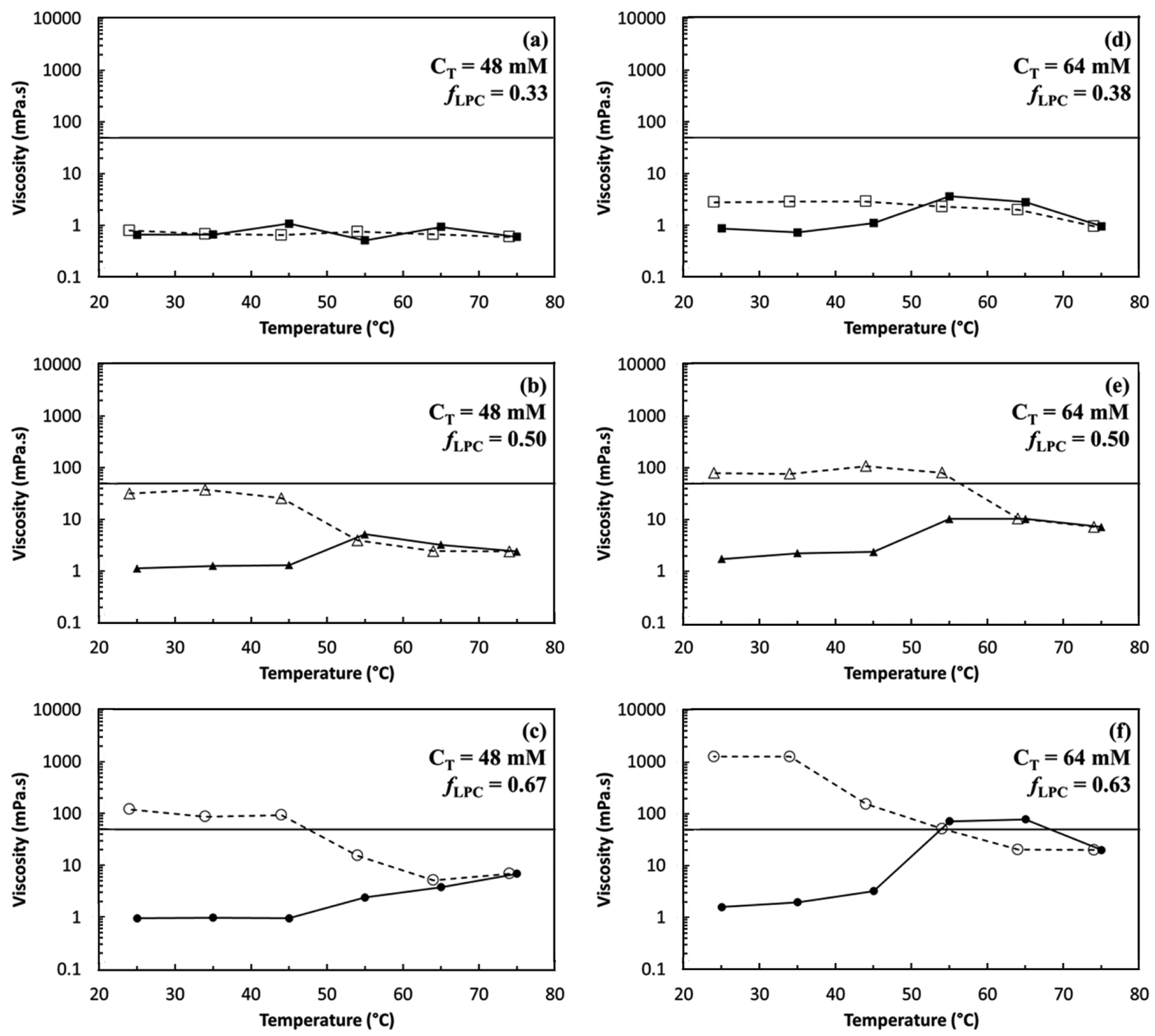

Fig. 5 Viscosity of $48 \mathrm{mM}(\mathrm{a}-\mathrm{c})$ and $64 \mathrm{mM}(\mathrm{d}-\mathrm{f})$ of lecithin mixtures $\left(f_{\mathrm{LPC}}\right)$ as a function of temperature. The molar fraction of LPC $\left(f_{\mathrm{LPC}}\right)$ is indicated in the figure. Filled symbols, heating; open symbols, cooling. Plots for cooling process were shifted by $-1^{\circ} \mathrm{C}$ for clarity. 
tively. Because the initial state of the mixtures is obviously not an equlibrium one, we observe first the increase in viscosity due to overcoming of a kinetic barrier. However, once the this kinetic barrier has been passed, increasing temperature is expected to decrease the viscosity, as explained above. Indeed, further heating above about $55^{\circ} \mathrm{C}$ led to a decrease of the viscosity, which we can assign to the effect of temperature on the equilibrium length of the wormlike micelles. Indeed, the viscosity of mixtures with $\mathrm{C}_{\mathrm{T}}=64 \mathrm{mM}$ also showed a marked increase on subsequent cooling below $60^{\circ} \mathrm{C}$.

Fig. 5 shows a clear hysteresis between the heating and cooling processes. It seems that heating is needed to overcome a certain kinetic barrier for formation of the wormlike micelles, but this structure is more stable than the mixture of individual planar and spherical micelles. The wormlike micelle is the dominant form at low temperature at equilibrium, which can be reached much more quickly by first heating the mixture to above $50^{\circ} \mathrm{C}$. Reorganization of the micelles probably requires enhanced mobility, which is attained at elevated temperatures. We do not wish to speculate on the exact nature of the kinetic barrier that needs to be overcome, but in comparison with single-surfactant micelles, it is clear that micelles formed by two types of surfactants should have a higher degree of internal order. The exact approach of the chains could therefore require a higher kinetic energy compared with that needed for formation of the native single-surfactant micelles. We note that formation of wormlike micelles on heating has also been reported by Tung et al. [4] and Shrestha et al. [33], who proposed that surfactant exchange was increased at elevated temperature, and this increased mobility facilitated formation of intermicellar interaction, resulting in formation of the wormlike micelles.

\subsection{Molecular mobility studied by NMR}

The diffusion coefficient $D$ of LPC and PC was measured using PFG-STE NMR. In spectra with $g>100 \mathrm{G} /$ $\mathrm{cm}$, several peaks were found between 0-6 ppm and assigned to protons on the glycerol backbone and the alkyl chain of fatty acids in LPC (Fig. 6), cf. peak assignment by Haque et al. [34]. The total peak area over this range (0-6 ppm, without the water peak) was used in the diffusion analysis.

Figure $7 \mathrm{a}$ shows the relative echo intensity $\left(\mathrm{I} / \mathrm{I}_{0}\right)$ as a function of $\gamma^{2} \delta^{2} g^{2}(\Delta-\delta / 3)$ of solutions of pure PC and LPC. In these samples, $\mathrm{I} / \mathrm{I}_{0}$ shows a single exponential decay. Therefore, the diffusion coefficient $D$ could be cal- culated from eq. (3) for all concentrations and temperatures tested of pure LPC or PC. This finding means that a narrow size distribution of micelles was present in all single-surfactant solutions.

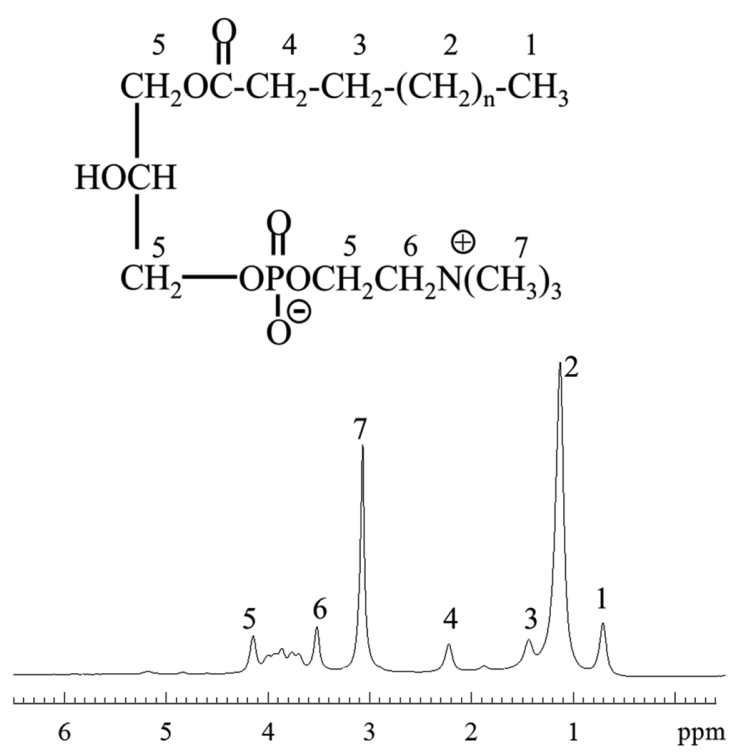

Fig. 6 Proton NMR Spectrum of $92.5 \mathrm{mM}$ lysophosphatidylcholine (LPC) in $\mathrm{D}_{2} \mathrm{O}$ at $25^{\circ} \mathrm{C}$

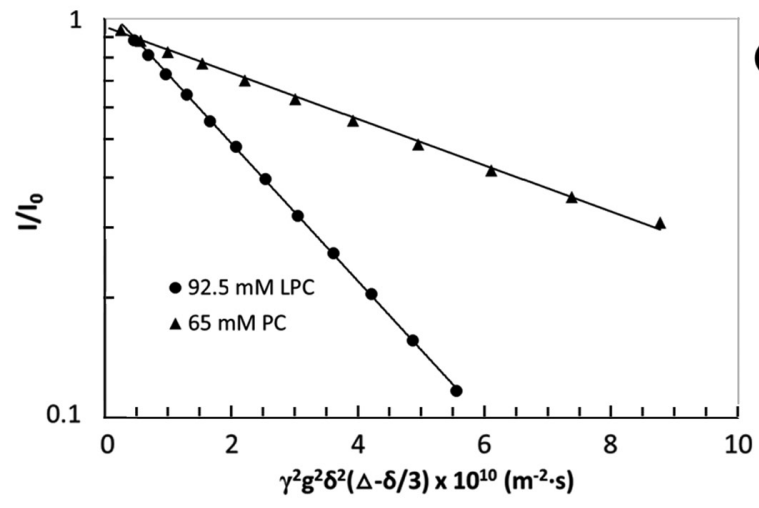

(a)

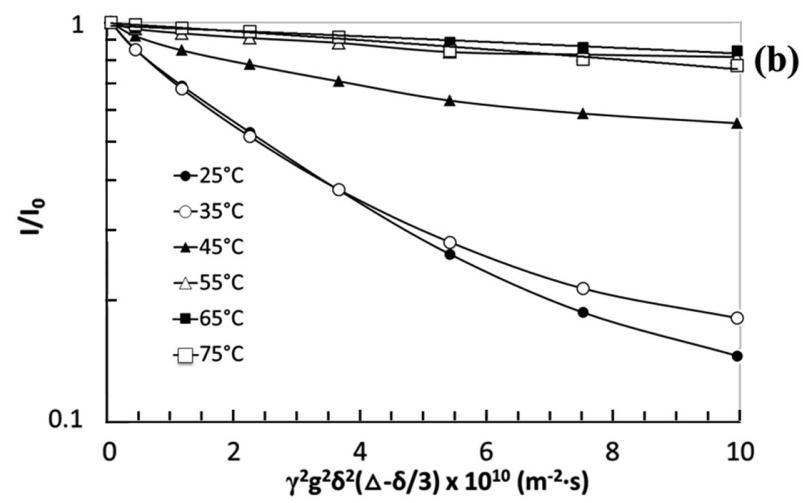

Fig. 7 Semi-logarithmic representation of the relative NMR echo intensity $\left(\mathrm{I} / \mathrm{I}_{0}\right)$ as a function of $\gamma^{2} \mathrm{~g}^{2} \delta^{2}(\triangle-\delta / 3)$ for (a) individual phospholipids at $25^{\circ} \mathrm{C}$ (a) and (b) a lecithin mixture $\left(\mathrm{C}_{\mathrm{T}}=78.75 \mathrm{mM}, f_{\mathrm{LPC}}=0.6\right)$ different temperatures indicated in the figure. Solid lines indicate least square fits to eq. (3) or eq. (4). 
The relative echo intensity $\left(\mathrm{I} / \mathrm{I}_{0}\right)$ in the $\mathrm{PFG}-\mathrm{STE}$ experiment for $\mathrm{C}_{\mathrm{T}}=64 \mathrm{mM}$ and $f_{\mathrm{LPC}}=0.6$ as a function of temperature is shown in Fig. $7 \mathrm{~b}$. At $25^{\circ} \mathrm{C}$ and $35^{\circ} \mathrm{C}$, a clear curvature is seen, which indicates the presence of diffusion of more than one component. If we assume the presence of two populations, we can rewrite equation (3) as follows:

$$
\begin{aligned}
I(\mathrm{~g})= & I(0)\left(f_{1} \exp \left[-\gamma^{2} \delta^{2} g^{2}(\Delta-\delta / 3) D_{1}\right]\right. \\
& \left.+f_{2} \exp \left[-\gamma^{2} \delta^{2} g^{2}(\Delta-\delta / 3) D_{2}\right]\right)
\end{aligned}
$$

where subscripts 1 and 2 refer to the first (faster) and second (slower) components, respectively, and $f_{1}$ and $f_{2}$ are their respective fractions. We assumed that the fast component $\left(f_{1}\right)$ corresponded in all cases to LPC micelles. We therefore fixed in the fitting procedure $D_{1}$ to $D$ measured for solutions of LPC. Thus, we used only 2 fit parameters: the diffusion coefficient and fraction of the slower component, $D_{2}$ and $f_{2}$, respectively (note that $f_{1}+f_{2}=1$ ). The slower component is assumed to reflect diffusion of both PC micelles and the wormlike micelles formed by co-micellization of LPC and PC. Good fits to the data at 25,35 and $45^{\circ} \mathrm{C}$ could be obtained using eq. (4). As the temperature is increased, the wormlike micelle is formed, as evidenced by the disappearance of the faster diffusing component, which started at around $45^{\circ} \mathrm{C}$, in agreement with the viscosity data in Fig. 5 . The plots for 55,65 and $75^{\circ} \mathrm{C}$ were almost linear indicating that the first component became negligibly small. For these temperatures, fits were obtained using eq. (3). At these temperature, only the slow component is present, so that $f_{2}=1$ and $D_{2}=D$.

Figure 8 shows the $D$ values of LPC (7a) and PC (7b). For $3.75 \mathrm{mM} \mathrm{LPC}, D$ at $25^{\circ} \mathrm{C}$ was $4.93 \times 10^{-11} \mathrm{~m}^{2} \mathrm{~s}^{-1}$, which corresponds to a displacement of $1 \mu \mathrm{m}$ during the diffusion time of $10 \mathrm{~ms}$. There are two possible modes of diffusion of surfactant chains. One is micelle translation. The other is intermicellar displacements of individual surfactant chains (see, e.g., 35). LPC is assumed to form a spherical micelle with a diameter close to the combined length of two $\mathrm{C}-18$ alkyl chains (the most common alkyl chain in our LPC sample). The projection of a $\mathrm{C}-\mathrm{C}$ bond along the axis of alkyl chains is approximately $0.12 \mathrm{~nm}$. The length of a C-18 alkyl chain is therefore $17 \times 0.12 \approx$ $2.0 \mathrm{~nm}$. Because the displacement of ${ }^{1} \mathrm{H}(1 \mu \mathrm{m})$ is much larger than the length of two $\mathrm{C}-18$ chains $(4 \mathrm{~nm})$, it reflects translation of the spherical micelle, rather than intermicellar movements.

We can further analyze data using the Einstein-Stokes relation:

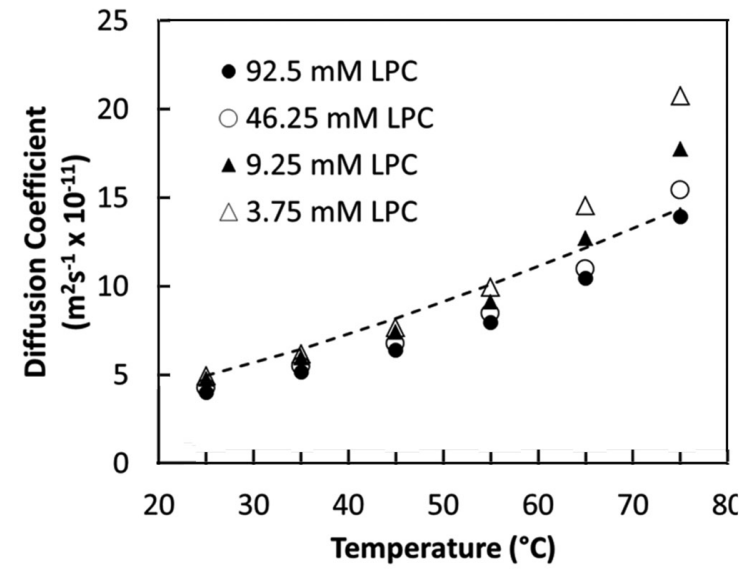

(a)

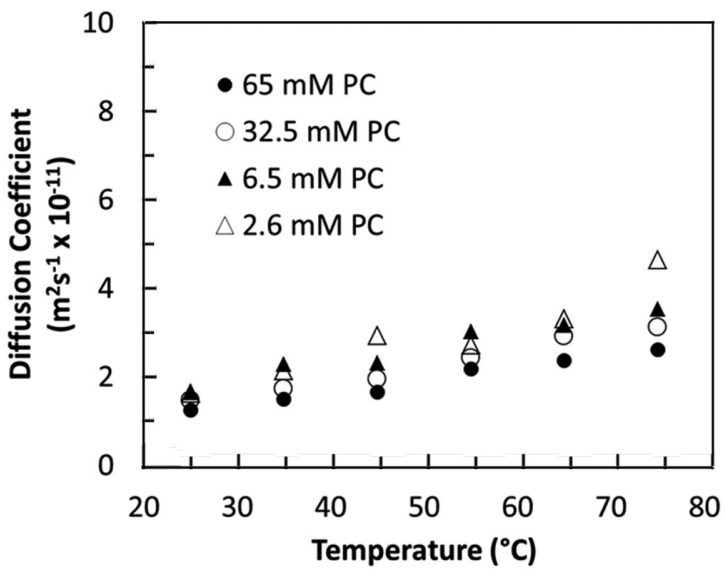

Fig. 8 Diffusion coefficient $D$ of lysophosphatidylcholine (LPC; a) and phosphatidylcholine (PC; b) in $\mathrm{D}_{2} \mathrm{O}$ as a function of temperature. The dashed line shows $D$ calculated using the Einstein-Stokes relation (eq. 5) assuming $R_{\mathrm{H}}=4 \mathrm{~nm}$.

$$
D=\frac{k_{\mathrm{B}} T}{6 \pi \eta R_{\mathrm{H}}}
$$

where $k_{\mathrm{B}}, T$ and $\eta$ are Boltzmann constant, absolute temperature and viscosity of the solvent, respectively. The hydrodynamic radius $R_{\mathrm{H}}$ is defined as the radius of the hard sphere which has the diffusion coefficient of $D$. Assuming that the LPC micelle is a hard sphere, we obtain $R_{\mathrm{H}}=4.98 \mathrm{~nm}$ for the $92.5 \mathrm{mM}$ solution at $25^{\circ} \mathrm{C}$. This value is a bit larger than the length of two alkyl chains. We expect this difference to reflect hydration of the LPC spheres. With increasing temperature, the $D$ value was increased, and the increase of $D$ seems to be steeper than that estimated from the increase of temperature. The diffusion coefficient of the spherical micelles formed by $3.75 \mathrm{mM}$ LPC solutions (calculated assuming a constant $R_{\mathrm{H}}$ value) is indicated with a dashed line in Fig. 8a. The $D$ value showed an upward deviation from this line at temperatures above $65^{\circ} \mathrm{C}$. As the temperature increases, the charge on phosphate choline head group decreases and some hydrogen bonding between the 
head groups and water is lost. As a result, the micelle size can decrease, as has been reported for SDS micelles in water [36].

The $D$ value of $2.6 \mathrm{mM} \mathrm{PC}$ at $25^{\circ} \mathrm{C}$ was $1.63 \times 10^{-11}$ $\mathrm{m}^{2} \mathrm{~s}^{-1}$, which corresponds to a displacement of $560 \mathrm{~nm}$ during the diffusion time of $10 \mathrm{~ms}$. Fig. 2 showed the formation of very large spherical PC micelles, which we assume to be vesicles with internal planar structure. Although it is probably only the largest vesicles which are observed, we still expect most PC assemblies to have diameters of at least several hundred $\mathrm{nm}$. A displacement of $560 \mathrm{~nm}$ could therefore reflect not only micelle displacement, but also intermicellar mobility of individual PC chains. PFG-NMR measures the self-diffusion coefficient. The self-diffusion coefficient of macromolecules in binary mixtures with a solvent always decreases with increasing macromolecule concentration, due to excluded-volume interactions and increased friction between the macromolecule and the solvent [37]. The micelle displacement should therefore be concentration dependent. On the other hand, the structure of most micelles is virtually independent of the surfactant concentration, and we therefore expect the intermicellar mobility to be independent of concentration of PC. Because we observe a decrease in $D$ with increasing concentration of PC, we can conclude that intermicellar displacements do not contribute significantly to the observed displacement of $560 \mathrm{~nm}$. Therefore, $D$ describes the movement of the PC micelle itself, and not (to any significant extent) that of individual PC molecules inside the micelle.

Figure 9 shows the slower component fraction $\left(f_{2}\right)$ and diffusion coefficient $\left(D_{2}\right)$ of a LPC/PC mixture $\left(f_{\mathrm{LPC}}=\right.$ $0.6)$ at various temperatures. The results obtained for all samples with $f_{\mathrm{LPC}}=0.6$ at $\mathrm{C}_{\mathrm{T}} \geq 39 \mathrm{mM}$ suggest the increase of wormlike micelle content with increasing temperature. This is reflected in the increase of $f_{2}$ and the decrease of $D_{2}$ values with increasing temperature up to $65^{\circ} \mathrm{C}$. Gokhale et al. [38] found a similar decrease in $D$ with increasing temperature due to the formation of wormlike micelle in CTAB with addition of $p$-nitrophenolate. Above $75^{\circ} \mathrm{C}, D_{2}$ increased with increasing temperature. This increase is similar to the increase seen for solutions of the individual surfactants as shown in Fig. 8. Similarly to our explanation of the viscosity data of Fig. 6, we can ascribe the reversing of the trend at high temperature to a decrease in the equilibrium length of the wormlike micelles. This effect can only be observed after the initial kinetic barrier to wormlike micelles formation
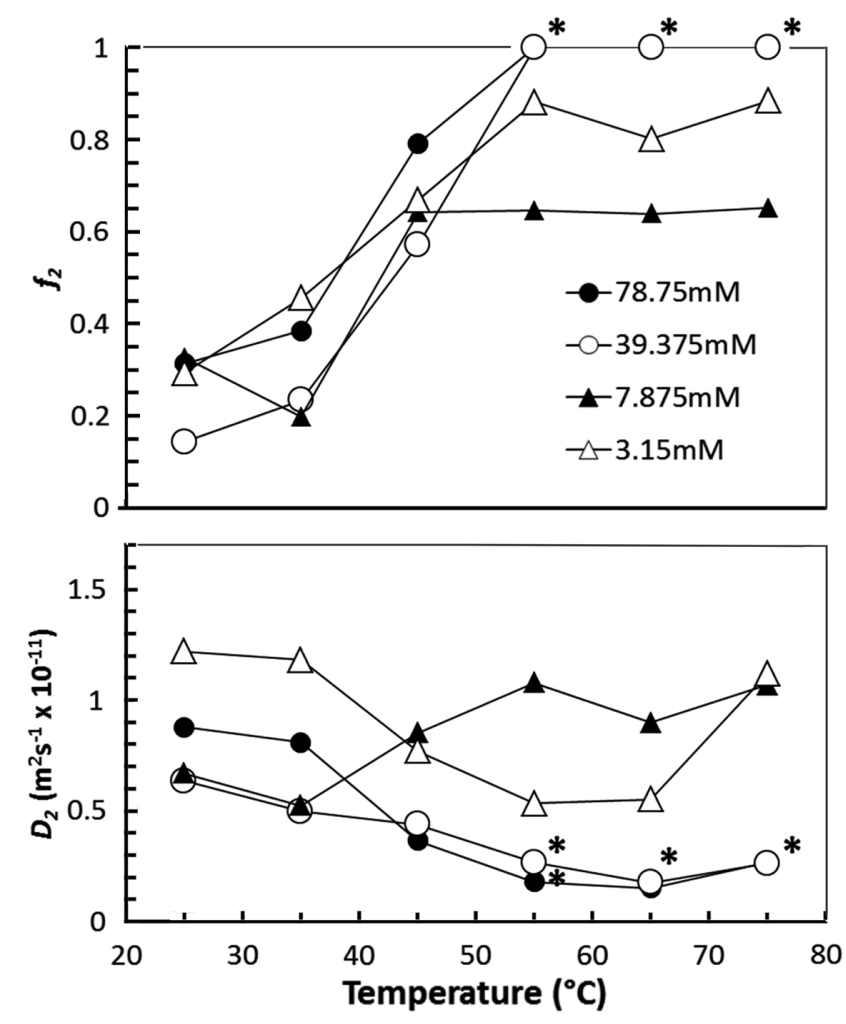

Fig. $9 f_{2}$ (Top) and $D_{2}$ (bottom) of lecithin mixtures with $f_{\mathrm{LPC}}$ $=0.6$ at various $\mathrm{C}_{\mathrm{T}}$ in $\mathrm{D}_{2} \mathrm{O}$ as a function of temperature. Measurements where a single diffusion component was found are indicated with an asterisk.

has been overcome.

It is clear from Fig. 9 that the increase in $f_{2}$ on heating of mixtures with $f_{\mathrm{LPC}}=0.6$ is also observed for mixtures at much lower total lecithin contents $\mathrm{C}_{\mathrm{T}}(3-8 \mathrm{mM})$. We assume that this increase also reflects formation of wormlike micelles. However, for these mixtures, $D_{2}$ shows unexpected behavior. We first note again that the equilibrium length of wormlike micelles is expected to decrease strongly with decreasing surfactant concentration [1]. It is therefore possible that the diffusion coefficient of the PC micelles, present in the initial non-equilibrium state, and the wormlike micelles which are formed on heating, is not so different. In addition, the diffusion of any PC micelles still present will be restricted in a non-trivial manner by the emerging wormlike structure. There is probably some compensation between the increase in restriction due to wormlike micelle formation and the increase in kinetic energy due to elevated temperature. It seems that these contrasting effects, along with the smaller difference between the size of the wormlike micelles and the PC micelles, can lead to either a decrease or increase in $D_{2}$ on heating of solutions with low $\mathrm{C}_{\mathrm{T}}$. 
It should be emphasized that the results showing two distinct populations with very different diffusion coefficients reflect a non-equilibrium state. As was already evidenced by the viscosity data shown in Fig. 5, the starting state at low temperature is not an equilibrium state. As the temperature increases, the kinetic barrier can be overcome, and the wormlike micelles are formed, as evidenced by the huge increase in viscosity. On cooling, the viscosity either remained close to its value at high temperature or increased. This finding indicates that the wormlike micelle is the thermodynamically stable state at low temperatures as well. This rheological hysteresis is a clear indication that the starting state at $25^{\circ} \mathrm{C}$ is not an equilibrium one. Therefore, the results shown in Fig. 9 should depend on the thermal history of the sample, as they reflect a non-equilibrium state during the first heating step. We note that several studies on formation of wormlike micelles from mixtures of cationic and anionic surfactants $[4,5]$ employed a methodology of initial extensive heating at temperatures ranging between $50-65^{\circ} \mathrm{C}$, and all characterization was done following this initial heating and assumed to be at an equilibrium state. Our investigation is different as we observed the changes in both surfactant diffusion and viscosity taking place on initial heating and wormlike micelle formation.

The information on phospholipid diffusion supports the rheological findings, namely, at $f_{\mathrm{LPC}} \approx 0.5-0.7$, wormlike micelles are formed on heating above $55^{\circ} \mathrm{C}$. In addition, it allows us to estimate the distribution of the two phospholipids between the small spherical micelles, which according to our results may very well contain a small amount of PC chains, and the larger planar and wormlike micelles. More importantly, the distribution indicates that at the highest temperatures tested, no fast diffusion component is left. This finding confirms that the wormlike micelles are formed by co-micellization of all the PC and LPC chains, and also means that no spherical micelles remain at the end of the co-micellization process.

\section{Conclusion}

Our research has demonstrated that a mixture of LPC and PC can form wormlike micelles in aqueous solution. Reverse wormlike micelle formation has been reported in the literature for phospholipids in w/o emulsions [3, $14,15]$, but this is the first report to our knowledge on formation of normal phospholipid wormlike micelles in water without the addition of salts. The formation of the wormlike micelle requires a minimum concentration of both PC and LPC. The data indicate that the wormlike micelle is more stable than the micelles formed by each phospholipid individually for all temperatures tested, but its formation requires heating in order to overcome kinetic barrier. Because uncharged phospholipids confer higher stability on $\mathrm{o} / \mathrm{w}$ emulsions compared to charged phospholipids when small amounts of $\mathrm{NaCl}$ or $\mathrm{CaCl}_{2}$ are present [26-28], the wormlike micelles reported herein could be used as both stabilizers and thickeners of such systems. We finally note that further research should be carried out to elucidate how general the formation of wormlike micelles is when two surfactants, one with $C P P$ $<0.5$ and the other with $C P P>0.5$, are mixed.

\section{References}

1) M. E. Cates, S. J. Candau; Statics and dynamics of worm-like surfactant micelles. J. Phys.: Condens. Matter, 2, 6869-6892 (1990).

2) H. Hoffmann; "Structure and Flow in Surfactant Solutions, ACS Symposium Series 578”, C. A. Herbs, R. K. Prud' homme (Eds.), American Chemical Society, 1994, pp. 2-31

3) R. Kumar, A. M. Ketner, S. R. Raghavan; Nonaqueous photorheological fluids based on light-responsive reverse wormlike micelles. Langmuir, 26, 5405-5411 (2009)

4) S. H. Tung, Y. E. Huang, S. R. Raghavan; Contrasting effects of temperature on the rheology of normal and reverse wormlike micelles. Langmuir, 23, 372-376 (2007).

5) T. S. Davies, A. M. Ketner, S. R. Raghavan; Self-assembly of surfactant vesicles that transform into viscoelastic wormlike micelles upon heating. J. Am. Chem. Soc., 128, 6669-6675 (2006).

6) Y. Lin, X. Han, J. Huang, H. Fu, C. Yu; A facile route to design pH-responsive viscoelastic wormlike micelles: Smart use of hydrotropes. J. Colloid Interface Sci., 330, 449-455 (2009).

7) M. J. Lawrence, G. D. Rees; Microemulsion-based media as novel drug delivery systems. Adv. Drug Delivery Rev., 45, 89-121 (2000).

8) A. Vintiloiu, J. C. Leroux; Organogels and their use in drug delivery - a review. J. Controlled Release., 125, 179-192 (2008).

9) J. Yang; Viscoelastic wormlike micelles and their applications. Curr. Opin. Colloid Interface Sci., 7, 276-281 (2002).

10) J. L. Blin, C. Gérardin, C. Carteret, L. Rodehüser, C. Selve, M. J. Stébé; Direct one-step immobilization of glucose oxidase in well-ordered mesostructured silica using a nonionic fluorinated surfactant. Chem. Mater., 17, 1479-1486 (2005).

11) F. Wang, X. Chen, Y. Xu, S. Hu, Z. Gao; Enhanced electron 
transfer for hemoglobin entrapped in a cationic gemini surfactant films on electrode and the fabrication of nitric oxide biosensor. Biosens. Bioelectron., 23, 176-182 (2007).

12) P. A. Hassa, B. S. Valaulikar, C. Manohar, F. Kern, L. Bourdieu, S. J. Candau; Vesicle to micelle transition: rheological investigations. Langmuir, 12, 4350-4357 (1996).

13) K. Horbaschek, H. Hoffmann, C. Thunig; Formation and properties of lamellar phases in systems of cationic surfactants and hydroxy-naphthoate. J. Colloid Interface Sci., 206, 439-456 (1998).

14) Y. A. Shchipunov; Lecithin organogel: a micellar system with unique properties. Colloids Surf., A, 183, 541-554 (2001).

15) E. V. Shumilina, Y. L. Khromova, Y. A. Shchipunov; The effect of lysophosphatidylcholine and phosphatidylglycerol on lecithin polymer-like micelles. Colloid J., 68, 241-247 (2006).

16) D. J. Hanahan; “A Guide to Phospholipid Chemistry”, Oxford University Press, 1997.

17) K. Chiba, M. Tada; Study of emulsion stability and headgroup motion of phosphatidylcholine and lysophophatidylcholine by ${ }^{13} \mathrm{C}$ - and ${ }^{31} \mathrm{P}-\mathrm{NMR}$, Agric. Biol. Chem., 53, 9951001 (1989).

18) M. Faergemand, N. Krog; "Texture in food. Volume 1: Semisolid foods”, B. M. McKenna ed., CRC Press, Boca Raton, 2003, pp. 216-250.

19) N. Krog; "Lipid technologies and applications", F. D. Gunstone, F. B. Padley (Eds.), Marcel Dekker, 1997, pp 521534 .

20) R. Pichot, R. L. Watson, I. T. Norton; Phospholipids at the Interface: Current Trends and Challenges. Int. J. Mol. Sci., 14, 11767-11794 (2013).

21) J. N. Israelachvili; "Intermolecular and surface forces" Third edition, Academic Press, 2011.

22) V. V. Kumar; Complementary molecular shapes and additivity of the packing parameter of lipids, Proc. Natl. Acad. Sci. USA, 88, 444-448 (1991)

23) W. S. Price, F. Tsuchiya, Y. Arata; Lysozyme aggregation and solution properties studied using PGSE NMR diffusion measurements. J. Am. Chem. Soc., 121, 11503-11512 (1999).

24) S. Gröger, D. Geschke, J. Kärger, F. Stallmach, Č. Koňák; Co - Micellization Investigated by Pulsed Field Gradient - NMR Spectroscopy. Macromol. Rapid Commun., 25, 1015-1018 (2004).

25) R. Lamanna; On the inversion of multicomponent NMR relaxation and diffusion decays in heterogeneous systems. Concepts Magn. Reson., Part A, 26, 78-90 (2005).

26) J. Han, C. Washington; Partition of antimicrobial additives in an intravenous emulsion and their effect on emulsion physical stability. Int. J. Pharm, 288, 263-271 (2005).

27) J. Han, S. S. Davis, C. Washington; Physical properties and stability of two emulsion formulations of propofol. Int. J. Pharm., 215, 207-220 (2001).

28) C. Washington, A. Athersuch, D. J. Kynoch; The electrokinetic properties of phospholipid stabilized fat emulsions. IV. The effect of glucose and of pH. Int. J. Pharm., 64, 217-222 (1990).

29) M. Brizard, M. Megharfi, E. Mahe, C. Verdie; Design of a high precision falling-ball viscometer. Rev. Sci. Instrum., 76, 025109 (2005).

30) W. S. Price; "Specialized PGSE and related techniques”, W. S. Price (Ed.), Cambridge University Press, New York, 2009, pp. 256-295

31) E. O. Stejskal, J. E. Tanner; Spin diffusion measurements: spin echoes in the presence of a time - dependent field gradient. J. Chem. Phys., 42, 288-292 (1965).

32) L. Arleth, R. Bauer, L. H. Øgendal, S. U. Egelhaaf, P. Schurtenberger, J. S. Pedersen; Growth behavior of mixed wormlike micelles: a small-angle scattering study of the lecithin-bile salt system. Langmuir, 19, 4096-4104 (2003).

33) R. G. Shrestha, L. K. Shrestha, K. Aramaki; Formation of wormlike micelle in a mixed amino-acid based anionic surfactant and cationic surfactant systems. J. Colloid Interface Sci., 311, 276-284 (2007).

34) R. Haque, I. J. Tinsley, D. Schmedding; Nuclear magnetic resonance studies of phospholipid micelles. J. Biol. Chem., 247, 157-161 (1972).

35) D. Myers; "Surfactant science and technology", John Wiley \& Sons, 2005

36) B. Hammouda; Temperature Effect on the Nanostructure of SDS Micelles in Water. J. Res. Natl. Inst. Stand. Technol., 118, 151-167 (2013).

37) C. Le Bon, T. Nicolai, M.E. Kuil, J.G. Hollander; SelfDiffusion and Cooperative Diffusion of Globular Proteins in Solution. J. Phys. Chem. B, 103, 10294-10299 (1999).

38) G. D. Gokhale, P. A. Hassan, S. D. Samant; Change in the aggregational character of the cationic surfactant cetyl trimethyl ammonium bromide in the presence of 0 -and p-itrophenolates. J. Surfactants Deterg., 8, 319-323 (2005). 